\title{
Addendum to: A Multifaceted Model of Changes and Adaptation among Korean Mothers of Children with Disabilities
}

\author{
Hye Jun Park · Grace H. Chung
}

(C) Springer Science+Business Media New York 2014

This Addendum supplements the author information for the article title "A Multifaceted Model of Changes and Adaptation among Korean Mothers of Children with Disabilities". Hye Jun Park is the first author and Grace H. Chung is the corresponding author. 